\title{
Relación entre el solto vertical y el rendimiento de la velocidad en jóvenes futbolistas
}

\section{Brian Johan Bustos-Viviescas ${ }^{1 *}$, Andrés Alonso Acevedo-Mindiola ${ }^{1}$ y Leidy Estefanía Rodríguez-Acuña'}

1 Estudiantes de la Licenciatura en Educación Básica con énfasis en Educación Física, Recreación y Deportes (Facultad de Educación, Departamento de Educación Física y Deporte, Universidad de Pamplona, Colombia)

Email: * brian.bustos@unipamplona.edu.co

RESUMEN: El objetivo de este estudio fue determinar la relación entre el salto vertical y el rendimiento de la velocidad en jóvenes futbolistas. Para lograr este objetivo se realizó un estudio descriptivo de tipo correlacional con enfoque cuantitativo y una muestra a conveniencia conformada por 30 jugadores (edad de $12.88 \pm 1.90$ años; peso corporal de $44.84 \pm 14.57 \mathrm{~kg}$; talla de $154.27 \pm 14.80 \mathrm{~cm}$; experiencia en el entrenamiento del fútbol de $4.09 \pm 1.81$ años) de la Escuela Talento Local Fútbol Club de la Ciudadela de Atalaya, Norte de Santander, Colombia. Las pruebas realizadas fueron el countermovement jump (CMJ) y sprint 20 metros (S20), la estimación de la altura del salto se efectuó por medio de análisis de video en el software Kinovea $\vee$. 0.7.10, mientras que para obtener la velocidad de carrera se utilizaron conos y cronómetro Casio Hs-80tw. Para el análisis de los datos se empleó el IBM SPSS V. 22 en el cual se determinaron las medias y desviaciones típicas, así como la relación entre el CMJ y el $\$ 20$ a través del coeficiente correlacional de Spearman. Los resultados obtenidos mostraron que la correlación entre el CMJ y el S20 fue negativa y significativa $(r=-.44 ; p<$ .05). En conclusión, la altura del salto vertical está relacionada con la velocidad de carrera en jóvenes futbolistas.

PALABRAS CLAVE: Evaluación deportiva, Fuerza, Deportistas, Rendimiento deportivo.

\section{Relationship between the vertical jump and the performance of speed in young soccer players}

ABSTRACT: T The objective of this study was to determine the relationship between the vertical jump and the performance of speed in young soccer players. To achieve this objective was a descriptive correlational study with a sample comprised 30 players convenience and quantitative approach lage of $12.88 \pm 1.90$ years; body weight of $44.84 \pm 14.57 \mathrm{~kg}$; size of $154.27 \pm 14.80 \mathrm{~cm}$; 
experience in the training of the soccer of $4.09 \pm 1.81$ years) of the school talent Local Soccer Club of the Citadel of Atalaya, North of Santander, Colombia. The tests performed were the countermovement jump (CMJ) and sprint 20 meters (S20), the estimate of the height of the jump was made software Kinovea $V$. 0.7.10 by video analysis, while for stroke rate is they used cones and stopwatch Casio Hs-80tw. IBM SPSS V. 22 was used for data analysis which determined the means and standard deviations, as well as the relationship between the CMJ and the S20 through Spearman correlation coefficient. The results showed that the correlation between the S20 and the CMJ was negative and significant ( $r=-$ .44; $p<.05)$. In conclusion, the vertical jump height is related to running speed in young soccer players.

KEY WORDS: Sports evaluation, Strength, Athletes, Sports performance.

\section{INTRODUCCIÓN}

El deporte de mayor influencia ha sido el fútbol a través de la historia (Mariño y Ortega, 2007), por lo que desde el punto de vista condicional entre los eventos más interesantes durante un partido de fútbol son representados por situaciones de alta intensidad tales como sprints y los saltos (Hoof y Helgerud, 2004), debido a que el desempeño competitivo en el fútbol depende de diferentes factores como la potencia muscular y la velocidad máxima alcanzada por los jugadores en distancias cortas (Stølen, Chamari, Castagna, y Wisloff, 2005). Cabe destacar que estos movimientos dentro del fútbol demandan altos niveles de fuerza rápida y potencia (Thomas, French, y Hayes, 2009), considerando que dentro de los deportes colectivos, cualquier acción implica acciones motoras dinámicas donde todos los jugadores tienen que alcanzar un elevado nivel de ejecución (Ferrando y Schneider, 2013), en vista de que existen situaciones de juego en las que realizar un salto o un sprint son decisivas en el resultado del partido (Stølen et al., 2005).

La velocidad es una exigencia técnica elemental para el rendimiento deportivo en el fútbol (Alanazi y Aouadi, 2015), en vista de que durante el desarrollo de un partido el jugador que tenga una buena aceleración podrá posicionarse antes que otro jugador a un balón dividido o en un contrataque o acción defensiva saldría vencedor (García y Peña, 2016). Por otro lado es de resaltar que, aunque los sprints no son las actividades más frecuentes durante un partido, si son un parámetro fundamental que determina el desempeño del jugador durante un partido (Milanovic et al., 2014), por ende, en muchos deporte individuales o de conjunto el sprint es un paramento importante del rendimiento deportivo (García y Peña, 2016).

Por otra parte, el salto permite en el deporte determinar ciertos niveles de potencia, coordinación, fuerza y velocidad (Rodríguez, Merchán, y Forero, 2014), es por ello que, en el fútbol el desempeño en movimientos explosivos como el salto vertical resulta de elevada importancia (Santos-García, López, Mallo, y Navarro, 2010), dado a que el rendimiento de un jugador puede verse incrementado con la fuerza de 
los miembros inferiores y con esto incrementar los resultados durante el encuentro (Piedrahita y Marin, 2014). Así mismo la velocidad de sprint y el salto contramovimiento (CMJ) están directamente relacionadas (Ramírez-Campillo et al., 2014), por lo que es fundamental evaluar la correlación existente entre la altura del salto vertical y el rendimiento de la velocidad, debido a que estos parámetros para los jóvenes futbolistas deben tenerse en cuenta dentro del proceso de planificación del entrenamiento (García-Pinillos, Ruiz-Ariza, Navarro-Martínez, y Latorre-Román, 2014).

Además la competitividad en el fútbol ha tenido una auge creciente que ha llevado a su vez a desarrollar investigaciones sobre los factores que permiten mejorar el éxito competitivo (Vallenilla y Gamardo, 2012), en vista de lo anterior resulta importante realizar evaluaciones físicas para realizar un control y seguimiento de la planificación del entrenamiento según los resultados obtenidos con los deportistas (Acevedo-Mindiola y Bustos-Viviescas, 2017), por lo tanto, el objetivo del presente estudio fue determinar la relación entre el salto vertical y el rendimiento de la velocidad en jóvenes futbolistas.

\section{MÉTODO}

\subsection{Diseño del estudio}

Estudio descriptivo de tipo correlacional con enfoque cuantitativo y muestra a conveniencia, así mismo al ser una investigación de campo se desarrolló con un diseño ex post facto.

\subsection{Participantes}

Los participantes fueron 30 jugadores (edad de 12.88 \pm 1.90 años; peso corporal de $44.84 \pm 14.57 \mathrm{~kg}$; talla de $154.27 \pm 14.80 \mathrm{~cm}$; experiencia en el entrenamiento del fútbol de 4.09 \pm 1.81 años) pertenecientes del Escuela Talento Local Fútbol Club de la Ciudadela de Atalaya, Norte de Santander, Colombia. Teniendo presente como criterios de inclusión para participar del estudio que se tuviera como mínimo 2 años de estar entrenando fútbol y como criterios de exclusión presentar una lesión o patología osteomuscular, metabólica o cardiorrespiratoria, al igual que tener alguna molestia o dolor durante la ejecución de los test físicos. Cabe destacar que todos los participantes firmaron un consentimiento informado por escrito en el cual declaraban su participación voluntaria y que los datos obtenidos en este estudio se utilizarían con fines de investigación, igualmente como se estaba trabajando con menores de edad este formato también fue firmado por los padres de familia de los deportistas.

Por otro lado, previo al desarrollo del estudio se contactó con los directivos del club, así como con los entrenadores para obtener su respectiva autorización. Además, este estudio considero los estándares internacionales expuestos en la Declaración de Helsinki (Asociación Médica Mundial, 2013), igualmente se tuvieron en cuenta los establecidos para investigaciones en ciencias del deporte y del ejercicio (Harriss y Atkinson, 2013). 


\subsection{Instrumentos}

Para la evaluación de la velocidad se empleó la prueba de sprint de 20 metros, debido a que otros estudios desarrollados anteriormente también han medido el tiempo en recorrer 20 metros a máxima velocidad en futbolistas (Alikhajeh, Ramezanpour, y Moghaddam, 2011; Comfort, Stewart, Bloom, y Clarkson, 2013; García-Pinillos, Ruiz-Ariza, Moreno, y Latorre-Román, 2015; García-Pinillos et al., 2014; Hernández, y García, 2012; Mathisen, y Danielsen, 2014, 2015; Ramos, 2010; Peñailillo, Espíldora, Jannas-Vela, Mujika, y Zbinden-Foncea, 2016), para el desarrollo de esta prueba se utilizaron conos y dos cronómetros Casio Hs-80tw.

Por otra parte, se efectuó el countermovement jump (CMJ) para el salto vertical, ya que es un parámetro utilizado en otras investigaciones con futbolistas (Castilla, Almagro, Arrayás, Fernández y Sáenz-López, 2014; Comfort et al., 2013; García-Pinillos et al., 2014; García-Pinillos et al., 2015), por ende, para la estimación de la altura del salto vertical se utilizó una cámara de video de 8 megapíxeles (1080p, 300 FPS), esta fue ubicada en vista sagital al participante, se iniciaba el video y el participante efectuaba el CMJ desde posición parado, posteriormente al retornar al suelo se finalizaba el video.

Además, se empleó el software Kinovea V. 0.8.15 para determinar el tiempo de vuelo (c/s) el cual corresponde al momento en el que el deportista pierde contacto con el suelo por el despegue y retorna a este cuando desciende del salto. A partir del tiempo de vuelo $\left(T_{\text {air }}\right)$ se puede obtener la velocidad vertical de despegue $(\mathrm{Vv})$ cuya ecuación se muestra a continuación: $V_{v}=1 / 2{ }^{*} T_{\text {air }}{ }^{*} g$ (donde $T_{\text {air }}$ es tiempo de vuelo representado en centisegundos).

Posteriormente se calculó la altura del salto (Hmax) en centímetros $(\mathrm{cm})$ con la siguiente ecuación: $H \max =\mathrm{V}_{\mathrm{v}}{ }^{2}{ }^{*}(2 \mathrm{~g}){ }^{-1}$

\subsection{Procedimientos}

Para el desarrollo del estudio se realizaron las evaluaciones en 3 días, el primero fue una simulación del test de salto CMJ y sprint de 20 metros para que los participantes se familiarizaran con el protocolo de las pruebas; el segundo día se realizó el test de salto CMJ y el tercer día la prueba de sprint de 20 metros. Cabe destacar que se realizaba dos intentos del salto CMJ y el sprint de 20 metros por cada deportista con un descanso de 2 a 3 minutos entre un intento y otro (Ferragut, Cortadellas, Arteaga, y Calbet, 2003; Vila et al., 2012), por lo que se utilizó para el análisis el mejor de los dos intentos en cada prueba.

Por otro lado, cabe resaltar que en el caso del test de sprint 20 metros se utilizaron dos cronómetros y se ubicaron dos evaluadores durante el desarrollo de esta prueba, por ende, el deportista al culminar la prueba obtenía dos tiempos captados por cada evaluador y se promediaba este tiempo para dar mayor precisión a cada intento.

Así mismo la separación entre los 3 días oscilaba entre las 48-72 horas para garantizar la recuperación adecuada de los participantes como se representa en la Figura 1. 


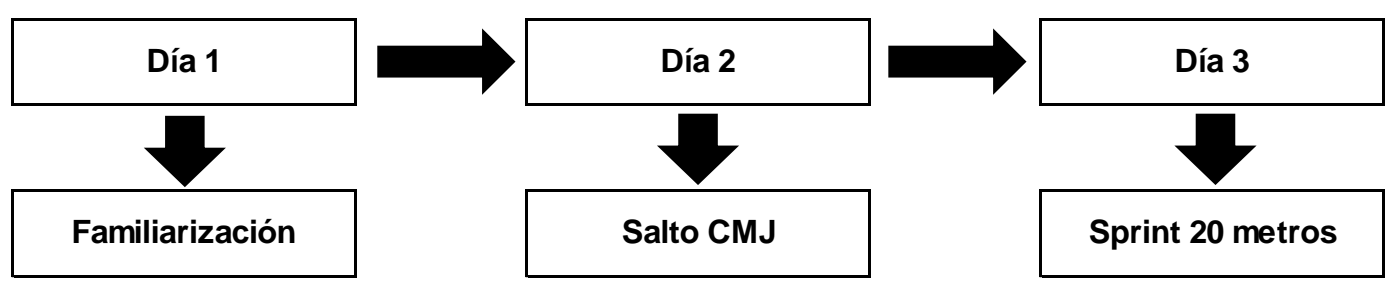

Figura 1. Distribución de las pruebas en los días establecidos

\subsection{Análisis de datos}

La determinación de las medias, desviaciones típicas y la altura del salto vertical se realizó en el Paquete estadístico IBM SPSS V. 22. Se aplicaron la prueba de normalidad de Shapiro-Wilk y el coeficiente correlacional de Spearman con un nivel de confianza del $95 \%$ y un p-valor de .05 .

\section{RESULTADOS}

\subsection{Características generales de los participantes}

En la Tabla 1 se pueden observar las medias y desviaciones estándar de la edad, talla, peso y experiencia en el entrenamiento del fútbol de los participantes.

Tabla 1. Características generales de los participantes

\begin{tabular}{ccccc}
\hline Jugadores $(\mathbf{n}=\mathbf{3 0})$ & Edad & Peso (kg) & Talla (cm) & $\begin{array}{c}\text { Experiencia en el entrenamiento } \\
\text { del fútbol (años) }\end{array}$ \\
\hline Media & 12.88 & 44.84 & 154.27 & 4.09 \\
Desviación estándar & 1.90 & 14.57 & 14.80 & 1.81 \\
\hline
\end{tabular}

\subsection{Normalidad de los datos}

Seguidamente en la Tabla 2 se pueden evidenciar los valores medios de altura del salto contramovimiento (CMJ) y el tiempo en la prueba sprint de 20 metros con su respectivo test de normalidad de Shapiro-Wilk. En la Tabla 2 y la Figura 2 se puede observar que el tiempo en la prueba sprint de 20 metros obtuvo una distribución normal ( $p>$.05). Sin embargo, la altura del salto CMJ no presentó una distribución normal $(p<.05)$, como se muestra en la Figura 3.

Tabla 2. Prueba de normalidad de los test aplicados en el estudio

\begin{tabular}{ccc}
\hline Jugadores $(\mathbf{n}=\mathbf{3 0})$ & Sprint 20 metros & Altura en salto CMJ \\
\hline $\begin{array}{c}\text { Prueba de Normalidad } \\
\text { (Shapiro-Wilk) }\end{array}$ & .53 & .01 \\
\hline
\end{tabular}




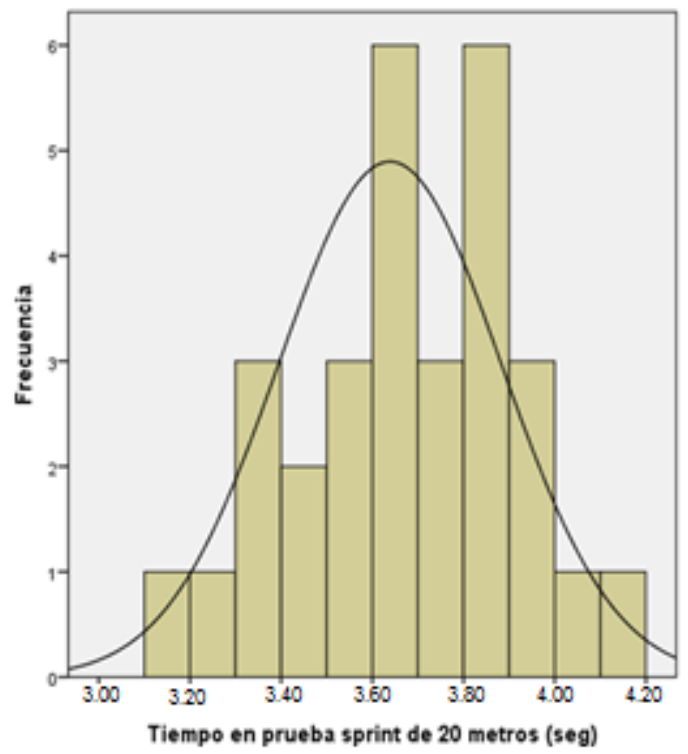

Figura 2. Histograma con curva normal de la prueba sprint de 20 metros

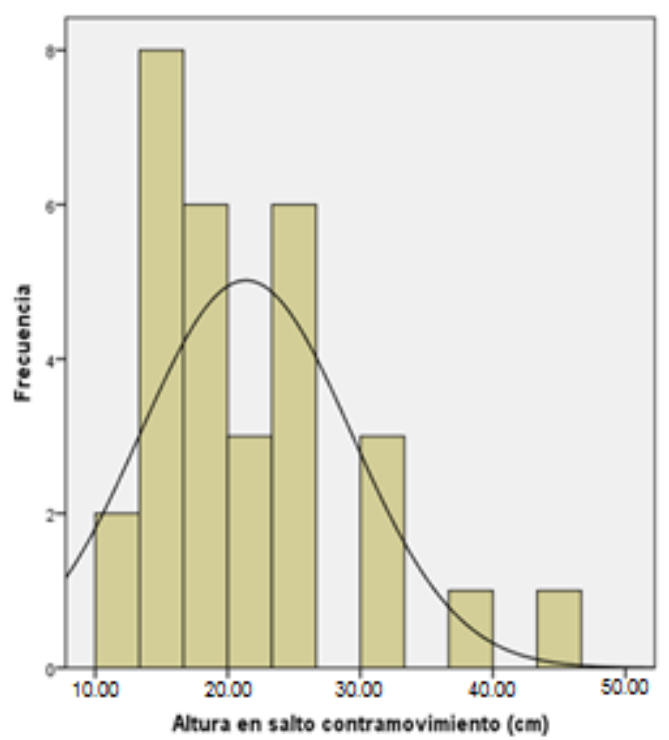

Figura 3. Histograma con curva normal del salto CMJ

\subsection{Relación entre el salto vertical y el sprint 20 metros}

En la Tabla 3 se presentan el coeficiente correlacional de Spearman con su respectivo nivel de significación entre los resultados de ambos test. La correlación obtenida fue negativa y significativa $(r=-.44 ; p<.05)$, por lo cual los participantes del estudio con menor altura en el salto CMJ tienden a tener menor rendimiento en la prueba sprint de 20 metros como se manifiesta en la Figura 4. 
Tabla 3. Coeficiente de correlación de Spearman

\begin{tabular}{cc}
\hline Jugadores $(\mathbf{n}=\mathbf{3 0})$ & $\begin{array}{c}\text { Sprint 20 metros-Altura en salto } \\
\text { CMJ }\end{array}$ \\
\hline $\begin{array}{c}\text { Coeficiente correlacional } \\
\text { de Spearman }\end{array}$ & -.44 \\
Significación (bilateral) & .02 \\
\hline
\end{tabular}

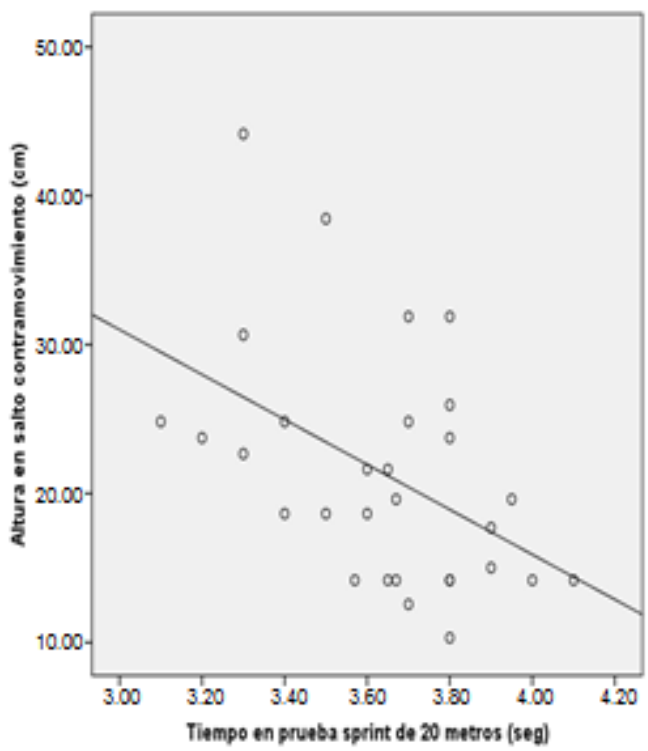

Figura 4. Gráfico de dispersión y línea de tendencia de los test

\section{DISCUSIÓN}

El objetivo del presente estudio fue determinar la relación entre el salto vertical y el rendimiento de la velocidad en jóvenes futbolistas. Entre los principales hallazgos de esta investigación se destaca que la relación existente entre estas dos variables es negativa y significativa $(r=-.44 ; p<.05)$, dado que los participantes que obtenían valores más elevados en el salto CMJ presentaban mejor rendimiento en la prueba sprint de 20 metros.

Estudios similares como el desarrollado por Frazilli, De Arruda, Mariano, y Cossio (2011) identifican la estrecha relación entre el CMJ y la velocidad del sprint de 20 metros en futbolista de 14 a 15 años $(r=-.61, p<.05)$. Igualmente, Irigoyen et al., (2014) encontraron una correlación negativa y muy significativa entre el salto horizontal con contramovimiento con manos libres y el sprint de 15 metros $(r=-.749, p$ <.01). Por otra parte, Köklüa, Alemdaroğlua, Özkanb, Kozc, y Ersöz (2015) obtuvieron 
esta misma tendencia entre el CMJ y el sprint de 30 metros $(r=-.599, p<.05)$. Sin embargo, los datos de estos estudios hacen referencia principalmente al fútbol de alto rendimiento o con deportistas de etapa adulta, por lo cual no se pueden extrapolar estos mismos resultados en aquellas poblaciones en etapa de crecimiento y desarrollo. En España, Castilla et al. (2014) en un estudio sobre la condición física y el índice de masa corporal en jugadores de fútbol en formación (entre 12 y 18 años), sí obtuvieron resultados similares a los obtenidos en esta investigación. De hecho, el coeficiente de correlación entre la velocidad en $15 \mathrm{~m}$ y el CMJ fue de $-.76(p<.01)$.

Según Asadi (2016) la asociación entre la capacidad de saltar (CMJ) y el rendimiento del sprint de 20 metros podría ser ocasionada por las necesidades de esfuerzo máximo al comenzar el sprint. En consecuencia es importante desarrollar altos niveles de la fuerza de miembros inferiores para mejorar el rendimiento del sprint y el salto en jugadores jóvenes de fútbol (Comfort et al., 2014).

Cabe destacar que en el fútbol el proceso de periodización y planificación al igual que otras modalidades deportivas tienen como objetivo principal la consecución de mejores resultados (Frazilli et al., 2011). Por ende es imprescindible estudiar aquellas variables que están estrechamente relacionadas con el rendimiento deportivo en etapas tempranas, esto con el fin de planificar las cargas del entrenamiento en estas poblaciones para que mejoren su forma física y obtengan altos logros en la etapa adulta. Entre estas variables de estudio se encuentran en el caso del fútbol aquellas relacionadas con la fuerza y la potencia muscular de miembros inferiores, por lo cual es necesario que se realicen más investigaciones sobre la relación entre la velocidad de sprint en línea recta y el salto vertical en jóvenes futbolistas.

Este estudio presentó algunas limitaciones. Los instrumentos empleados para la toma de datos (cronómetro, cámara de video y software Kinovea) se utilizaron como medios alternativos para alcanzar el objetivo de esta investigación, dado a que en el caso de la prueba de sprint no se contó con fotocélulas, así mismo no se tuvo la posibilidad de emplear una plataforma de salto, ni tampoco se tenía disposición de un dispositivo que contara con sistema operativo IOS 9 o superior para emplear la app MyJump validada para evaluar el salto vertical (Balsalobre-Fernández, Glaister, y Lockey, 2015). No obstante, la metodología empleada en este estudio se realizó con el propósito de obtener la mayor precisión posible en la recolección de los datos.

Se sugiere para futuros estudios que se incluyan variables como la flexibilidad de la musculatura isquiosural, la agilidad y el perfil antropométrico para establecer la relación con la velocidad y la altura del salto vertical, dado que las relaciones entre estos componentes del rendimiento en deportes colectivos no están del todo claras en etapas tempranas.

\section{CONCLUSIÓN}

A partir de los resultados evidenciados en este estudio se concluye que: la altura del salto vertical está relacionada con el rendimiento de carrera en jóvenes futbolistas. Por tal motivo, es fundamental incorporar programas de entrenamiento de 
fuerza y pliometría que permitan incrementar la altura del salto vertical para obtener mejor rendimiento de la velocidad en jóvenes futbolistas.

\section{REFERENCIAS}

Asadi, A. (2016). Relationship Between Jumping Ability,Agility and Sprint Performance of Elite Young Basketball Players: A Field-Test Approach. Revista Brasileira de Cineantropometria e Desempenho Humano, 18(2), 177-186 doi: http://dx.doi.org/10.5007/1980-0037.2016v18n2p177

Asociación Médica Mundial (2013). Declaración de Helsinki de la AMM-Principios éticos para las investigaciones médicas en seres humanos. Recuperado de http://www.isciii.es/ISCIII/es/contenidos/fd-investigacion/fd-evaluacion/fd-evaluacionetica-investigacion/Declaracion-Helsinki-2013-Esp.pdf

Acevedo-Mindiola, A. A., y Bustos-Viviescas, B. J. (2017). Correlación entre la flexibilidad de la musculatura isquiosural con la altura del salto vertical en jugadores de balonmano selección del departamento Norte de Santander. EDU-FÍSICA: Revista de Ciencias Aplicadas al Deporte, 9(20), 109-120. Recuperado de http://revistas.ut.edu.co/index.php/edufisica/article/view/1198/957

Alanazi, H. M., y Aouadi, R. (2015). Reaction time as a predictor for change-of-direction speed in male soccer players. Saudi Journal of Sports, 15(3), 220-225. doi: 10.4103/13196308.164287

Alikhajeh, Y., Ramezanpour, M. R., y Moghaddam, A. (2011). The Effect of Different Warm-up Protocols on young Soccer Players' sprint. Procedia - Social and Behavioral Sciences, 30, 1588-1592, doi: https://doi.org/10.1016/i.sbspro.2011.10.308.

Balsalobre-Fernández C., Glaister, M., y Lockey, R. A. (2015). The validity and reliability of an iPhone app for measuring vertical jump performance. Journal of Sports Science, 33(15),1574-1579. doi: 10.1080/02640414.2014.996184

Castilla, J. B., Almagro, B. J., Arrayás, M. J., Fernández, E. J., y Sáenz-López, P. (2014). Condición física e índice de masa corporal en las categorías de formación de un club de fútbol. Habilidad Motriz, 42, 16-27. Recuperado de https://www.researchgate.net/profile/Eduardo Fernandez-

Ozcorta/publication/273122188 Condicion fisica e indice de masa corporal en las categorias de formacion de un club de futbol/links/5502133d0cf2d60c0e62994d.pdf

Comfort, P., Stewart, A., Bloom, L., y Clarkson, B. (2013). Relationships Between Strength, Sprint, and Jump Performance in Well-Trained Youth Soccer Players. The Journal of Strength and Conditioning Research 28(1). Recuperado de: https://www.researchgate.net/publication/236094216 Relationships Between Strength Sprint and Jump Performance in Well-Trained Youth Soccer Players

Comfort, P., Stewart, A., Bloom, L., y Clarkson, B. (2014). Relationships Between Strength, Sprint, and Jump Performance in Well-Trained Youth Soccer Players. Journal of Strength \& Conditioning Research, 28(1), 173-177. doi: 10.1519/JSC.0b013e318291b8c7

Frazilli, E. H., De Arruda, M., Mariano, T., y Cossio, M. A. (2011). Correlación entre fuerza explosiva y velocidad en jóvenes futbolistas. Biomecánica, 19(1), 19-24. Recuperado de https://upcommons.upc.edu/bitstream/handle/2099/12308/19-24.pdf 
Ferragut, C., Cortadellas, J., Arteaga, R., y Calbet, J. (2003). Predicción de la altura de salto vertical, importancia del impulso mecánico de la masa muscular de las extremidades inferiores. European Journal of Human Movement, 10, 7-22. Recuperado de http://dialnet.unirioja.es/servlet/oaiart?codigo $=2279024$

Ferrando, J. y Schneider, J. L. (2013). Relación entre el CMJ y cambios de dirección en deportes colectivos. Revista Iberoamericana de Ciencias de la Actividad Física y el Deporte, 2 (1), 30-35. Recuperado de http://www.riccafd.uma.es/DOCUMENTOS/articulos/VOL002/N1/SchneiderCambDirDxt sCols.pdf

García-Pinillos, F., Ruiz-Ariza, A., Navarro-Martínez, A., y Latorre-Román, P. A. (2014). Análisis del rendimiento en salto vertical, agilidad, velocidad y velocidad de golpeo en jóvenes futbolistas: influencia de la edad. Apunts: Medicine de L'Esport, 49(183), 67-73. doi: 10.1016/j.apunts.2014.05.002

García-Pinillos, F., Ruiz-Ariza, A., Moreno, R., y Latorre-Román, P. A. (2015). Impact of limited hamstring flexibility on vertical jump, kicking speed, sprint, and agility in young football players. Journal of Sports Sciences, 33(12). doi: https://doi.org/10.1080/02640414.2015.1022577

García Ramos, J., y Peña López, J. (2016). Efectos de 8 Semanas de Entrenamiento Pliométrico y Entrenamiento Resistido Mediante Trineo en el Rendimiento de Salto Vertical y Esprint en Futbolistas Amateurs. Kronos, 15(2). Recuperado de http://gse.com/es/entrenamiento-de-la-velocidad-y-agilidad/articulos/efectos-de-8-semanasde-entrenamiento-pliometrico-y-entrenamiento-resistido-mediante-trineo-en-elrendimiento-de-salto-vertical-y-esprint-en-futbolistas-amateurs-2192

Harriss, D. J., y Atkinson, G. (2013). Ethical standards in sport and exercise science research: 2014 update. International Journal of Sports Medicine, 34(12), 1025-1028. doi: http://dx.doi.org/10.1055/s-0033-1358756

Hernández, Y., y García, J. (2012). Efectos de un entrenamiento específico de potencia aplicado a futbolistas juveniles para la mejora de la velocidad lineal. Motricidad. European Journal of Human Movement, 28, 125-134. Recuperado de https://ruidera.uclm.es/xmlui/handle/10578/2900

Hoff, J., y Helgerud, J. (2004). Endurance and strength training for soccer players. Physiological considerations. Sports Medicine, 34, 165-180.

Irigoyen, J. Y., García Huerta, A., Castillo Alvira, D., Rivero Benito, L. Á., y Los Arcos Larumbe, A. (2014). Evaluación y relación entre distintos parámetros de condición física en futbolistas semi profesionales. RETOS: Nuevas Tendencias en Educación Física, Deporte y Recreación, 26, 114-117. Recuperado de http://www.redalyc.org/articulo.oa?id=345732292020

Köklüa, Y., Alemdaroğlua, U., Özkanb, A., Kozc, M., y Ersöz, G. (2015). The relationship between sprint ability, agility and vertical jump performance in young soccer players. Science \& Sports, 30(1), e1-e5. doi: https://doi.org/10.1016/..scispo.2013.04.006

Mariño, N. A., y Ortega, A. J. (2007). Cuantificación y evolución de la saltabilidad en niños practicantes de fútbol de campo. Lecturas: Educación Física y Deportes, Revista Digital, 112. Recuperado de http://www.efdeportes.com/efd112/saltabilidad-en-ninospracticantes-de-futbol-de-campo.htm

Mathisen, G. E., y Danielsen, K. H. (2014). Effects of speed exercises on acceleration and agility performance in 13-year-old female soccer players. Journal of Physical Education 
and Sport, 14(4), 471-474. Recuperado de https://munin.uit.no/bitstream/handle/10037/7395/article.pdf?sequence=1

Mathisen, G. E., y Danielsen, K. H. (2015). The effect of speed training on sprint and agility performance in female youth soccer players. Journal of Physical Education and Sport, 15(3), 395-399. Recuperado de https://search.proquest.com/openview/dd461ea5bc61c5d5b4414c1652c9db2f/1?pqorigsite $=$ gscholar $\& \mathrm{cbl}=1006394$

Milanovic, Z., Sporis, G., Traikovic, N., Sekulic, D., James, N., y Vuckovic, G. (2014). Does SAQ training improve the speed and flexibility of young soccer players? A randomized controlled trial. Human Movement Science, 38, 197-208.

Peñailillo, L., Espíldora, F., Jannas-Vela, S., Mujika, I., y Zbinden-Foncea, H. (2016). Muscle strength and speed performance in youth soccer players. Journal of Human Kinetics, 50, 203-210. doi: http://doi.org/10.1515/hukin-2015-0157

Piedrahita, O. D., y Marin, M. (2014). Como influye un plan de entrenamiento pliometrico en el salto vertical de los jugadores centrales y delanteros de la categoría sub 13-14 años del Club Inem del poblado, futbol masculino (Tesis de pregrado). Universidad de Antioquia, Medellín. Recuperado de http://viref.udea.edu.co/contenido/pdf/203-comoinfluye.pdf

Ramos, J. A. (2010). Características morfo-funcionales y motoras en jóvenes futbolistas como criterio de orientación y selección deportiva. Revista Educación física y deporte, 29(1), 45-54.

Recuperado

de http://aprendeenlinea.udea.edu.co/revistas/index.php/educacionfisicaydeporte/article/vi $\underline{\mathrm{ew} / 7160 / 6607}$

Ramirez-Campillo, R., Henríquez-Olguín, C., Burgos, C., Andrade, D., Zapata, D., Martinez, C., Baez, E. I., Castro-Sepulveda, M., Peñailillo, L., y Izquierdo, M. (2014). Effect of progressive volumen-based overload during plyometric training on explosive and endurance performance in young soccer players. Journal of Strength and Conditioning Research, 29(7), 1884-1893. doi: 10.1519/JSC.0000000000000836

Rodríguez, G. A., Merchan, J. A., y Forero, S. A. (2014). Comportamiento de la fuerza explosiva, la agilidad y la velocidad ante un calentamiento con sobrecarga en futbolistas. Revista de Entrenamiento Deportivo, 28(4). Recuperado de http://gse.com/es/entrenamiento-en-futbol/articulos/comportamiento-de-la-fuerza-explosiva-laagilidad-y-la-velocidad-ante-un-calentamiento-con-sobrecarga-en-futbolistas-1762

Santos-García, D. J., López, C., Mallo, J., y Navarro, E. (2010). Análisis del golpeo de balón y su relación con el salto vertical en futbolistas juveniles de alto nivel. Revista Internacional de Ciencias del Deporte, 6(19), 128-140. doi:10.5232/ricyde2010.01903

Stølen, T., Chamari, K., Castagna, C., y Wisløff, U. (2005). Physiology of soccer: An update. Sports Medicine, 35(6), 501-536.

Thomas, K., French, D., y Hayes, P. R. (2009). The effect of two plyometric training techniques on muscular power and agility in youth soccer players. Journal of Strength and Conditioning Research, 23, 332-335. doi: 10.1519/JSC.0b013e318183a01a

Vallenilla, M. J., y Gamardo, P. F. (2012). Potencia anaeróbica máxima en futbolistas de categorías menores del Distrito Capital. Lecturas: Educación Física y Deportes, Revista Digital, 175. Recuperado de http://www.efdeportes.com/efd175/potencia-anaerobicamaxima-en-futbolistas.htm

Vila, H., Manchado, C., Rodriguez, N., Abraldes, J. A., Alcaraz, P. E., y Ferragut, C. (2012). Anthropometric profile, vertical jump, and throwing velocity in elite female handball 
players by playing positions. The Journal of Strength and Conditioning Research, 26(8), 2146-2155. doi: 10.1519/JSC.0b013e31823b0a46

\section{Agradecimientos}

Los autores manifiestan sus agradecimientos a los jugadores, padres de familia, entrenadores y directivos de la Escuela Talento Local Fútbol Club de la Ciudadela de Atalaya por permitir el desarrollo de este estudio, igualmente a la Universidad de Pamplona por apoyar en esta investigación. 